\title{
Triboelectric generator using mesoporous polydimethylsiloxane and gold layer
}

\author{
Mariatul Rawdhah Ahmad Fuaad ${ }^{1}$, Lee Chang Yang ${ }^{2}$, Farah Afiqa Mohd Ghazali ${ }^{3}$, \\ Mohamed Sultan Mohamed $\mathrm{Ali}^{4}$ \\ ${ }^{1,3,4}$ School of Electrical Engineering, Universiti Teknologi Malaysia, Malaysia \\ ${ }^{2}$ NACT Engineering, Singapore
}

\section{Article Info}

Article history:

Received Nov 3, 2019

Revised Feb 3, 2020

Accepted Mar 15, 2020

\section{Keywords:}

Energy harvesting

Microgenerator

Triboeletric

\begin{abstract}
This paper presents a triboelectric generator using mesoporous (PDMS) polydimethylsiloxane and gold layer which was demonstrated in energy harvesting applications. The performance of power generation by the means of triboelectric principle at a small dimension, namely triboelectric generator is characterized. In this paper, triboelectric generator device adapted vertical contact-separation operation mode, whereby the device derives power generation based on contact electrification caused by cyclic tapping motion. Being primarily a two-layer structure, this device comprises a top layer of aluminum (Al) electrode coated with mesoporous polydimethylsiloxane (PDMS) film and another bottom layer of $\mathrm{Al}$ electrode coated with gold (Au) deposit. The characterization of this device is done by varying frequencies and cyclic compression force applied to triboelectric generator. The optimal performance of the $2 \mathrm{~cm} \times 2 \mathrm{~cm}$ triboelectric generator contact surface area generated an open-circuit voltage of $4.4 \mathrm{~V}$ and a current of $0.1 \mu \mathrm{A}$ at $5 \mathrm{~Hz}$ frequency. This research and device can be improved by magnifying the effective surface area of triboelectric generator to generate significant power for small base area.
\end{abstract}

This is an open access article under the CC BY-SA license.

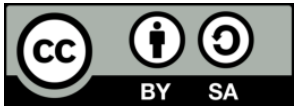

Corresponding Author:

Mohamed Sultan Mohamed Ali,

School of Electrical Engineering,

Universiti Teknologi Malaysia,

81310 UTM Johor Bahru, Johor, Malaysia.

Email: sultan_ali@fke.utm.my

\section{INTRODUCTION}

Triboelectric generator (TEG) is one of renewable energy latest technology for the depleting energy source such as coal, petroleum and natural gas. Due to high demand from rising population, renewable energy is a must to attain optimum accommodation. Although biomass, wind, hydroelectric and solar has been implemented however it was recently found that energy can be derived from a small activity scale and yet will produce a large quantity in its occurrence. Thus, various generators from five main energy harvesting technique appear such as electromagnetic, piezoelectric, pyroelectric, thermoelectric, and triboelectric. These generators carry specific attribute that can be used in their individual prerequisite applications.

In electromagnetic energy harvesting technique, electromagnetic induction generator (EMIG) is derived from a phenomenon which occurred when electrodynamic potential is induced across conductor rod when it moves across magnetic induction lines [1], given in (1):

$$
E=B \cdot l \cdot v
$$


Where $B$ is the density of magnetic flux, $l$ is the conductor rod length and $v$ is the conductor rod velocity cutting the magnetic induction lines. According to study [2], when the angular velocity is set at $20 \pi \mathrm{rad} / \mathrm{s}$, the rotating electromagnetic induction generator (EMIG) with 12 coil turns and magnetic flux density of $0.05 \mathrm{~T}$ induced $95.3 \mathrm{mV}$ of peak voltage. The output performance of rotating EMIG changes at different outer resistive load. However, the increased value in resistance results in an increment of the output voltage, although the output current will decline.

Piezoelectric energy harvesting technique is based on piezoelectric effect that is incorporating to certain material's ability to produce an electric charge out of response towards mechanical stress applied to it. Since the idea of harvesting piezoelectric energy was first proposed in the term piezoelectric nanogenerator (PENG) in 2006 [3], researchers prompted to implement PENGs for powering mobile devices by implementing different designs [4]. There have been several reports done to measure the performance of zinc oxide $(\mathrm{ZnO})$ nanogenerator which has both piezoelectric and semiconducting properties [5]. In a more recent research, a flexible piezoelectric ultrasonic energy harvester array was developed by incorporating a vast number of piezoelectric active elements into the elastomer membrane [6]. Due to its mechanism structure, PENG is able to generate voltage by minimal physical motions and large excitation frequency range. This allows PENG to harvest various environmental energy sources such as from microscopic vibrations, gentle air flow and also body motion. Nonetheless, PENG device is fragile and required a high cost to fabricate [7].

As for pyroelectric energy harvesting technique, the concept comes from the ability of certain materials in electrical potential generation when heated or cooled. Meaning electricity is derived from the production of charge in a stain-free condition, which overlooks the pyroelectric response in lead zirconate titanate (PZT) and certain other ferroelectric materials [8]. Pyroelectric generator primarily consists of three layers. Under a constant temperature, the overall mean strength of spontaneous polarization of the electric charges pair in nanowire-composite film is fixed, thus resulting in no electric output. When temperature increases, the pair of electric charges will oscillate with a larger range and this will cause a decrease in total average spontaneous polarization resulting in a reduction induced charges amount in electrodes and promote the flow of electrons through external circuit.

Thermoelectric energy harvesting technique has a very high potential to transform wasteful thermal energy into electricity attributable to renewable energy sources such as human body heat, biomass waste steam storage and hot water vapor $[9,10]$. Thermoelectric generator is a solid state device that converts temperature differences into electrical energy as a result of the phenomenon known as the Seebeck effect [11, 12]. Thermoelectric generator have several features such as high reliability and low cost performance, no maintenance required, and direct conversion without an intermediary energy conversion phase [13]. Previously research has shows thermoelectric generators can be fabricated using copper-cobalt $(\mathrm{Cu}-\mathrm{Co})$ [14] as positive and negative electrode respectively. However, some limitations found in the thermoelectric generator are including low power factor, very low thickness of thermoelectric material and high contact resistance [15].

Similar to other generators, triboelectric energy harvesting technique, as shown in Figure 1, is derived from the principle from what its name implies, triboelectric effect, which will transduce mechanical energy into electrical energy, based on touch electrification and electrostatic induction. The self-powered sensor can produce an electrical signal on its own, responding to stimuli from the environment without additional energy supply devices [16]. Hence, the potential of TEG as an energy harvester proved to be more efficient than other harvesting techniques such as electromagnetism, piezoelectricity and pyroelectricity.

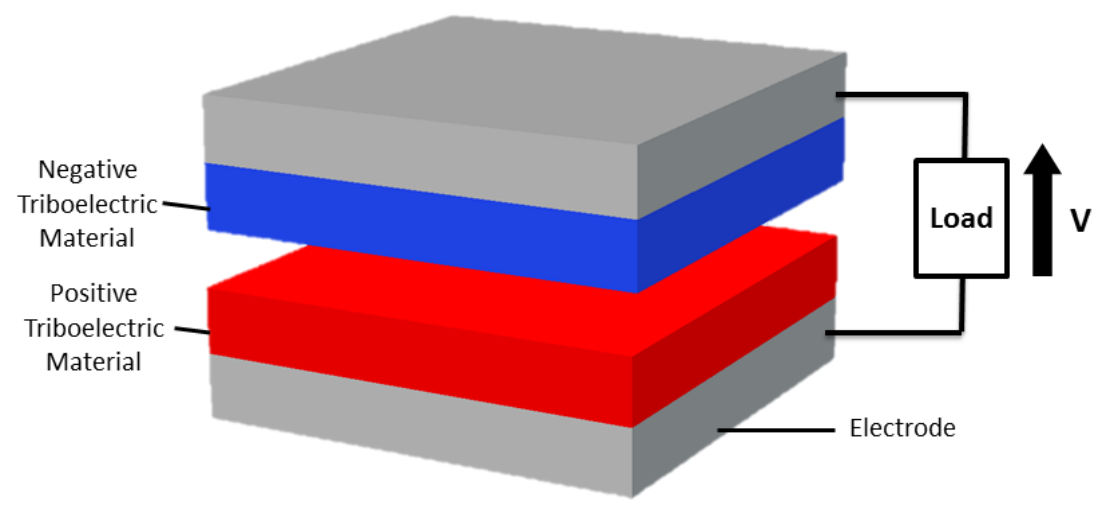

Figure 1. Triboelectric generator (TEG) 
Table 1 shown that amidst other energy harvesting techniques, TEG have depicted multiple advantages such as high power density, high voltage, and high efficiency at low frequency, low weight, low cost, various operation modes and choices of material. Therefore, it is a more ideal system to harvest electricity. It is found that there are four different operation modes for TEG, which are vertical contact separation mode [21], lateral sliding mode [22], single electrode mode [23] and freestanding triboelectric layer mode [24]. Vertical contact mode and lateral sliding mode are simple structures whereas single electrode mode and freestanding triboelectric layer mode are complex structures. Moreover, only vertical contact mode harvests energy from cyclic vertical tapping motion whereas the other operation modes derive energy from cyclic horizontal sliding. Performance-wise, all operation modes have high power output with lateral sliding mode being the highest followed by vertical contact mode, freestanding triboelectric layer mode and lastly, single electrode mode. By considering high power generation and long lifetime factors, vertical contact mode is the most suitable operation mode since it has the highest power output after lateral sliding mode and not vulnerable to wear and tear.

All of the modes generate electricity derived from electrostatic charges production by triboelectrification, in which the separation of electrostatic charges by friction induces current between two objects. A lot of attempts had been done by researchers on TEG and the issue is usually the lack of significant power generation, given that the contact surface area for dielectric material is limited, in order to accommodate the small and light-weight portable devices. This is where this research becomes essential to determine the adequacy of TEG to power up low energy portable devices, such that the TEG can supply a significant amount of power to the portable devices, yet meeting the requirements of the TEG system properties to be small in dimension as well as light in weight.

In this paper, the integrated approach of TEG has been demonstrated. The characteristic power output of the TEG has been acquired by varying the frequency and cyclic force applied between positive and negative electrode of TEG. This research will have focused on triboelectric energy harvesting techniques using mesoporous PDMS and Au coated.

Table 1. Comparison between four energy harvesting techniques [17-20]

\begin{tabular}{llllll}
\hline Description & Electro-magnetic & Piezoelectric & Pyroelectric & Thermoelectric & Triboelectric \\
\hline Characteristics & Complex structure & Fragile & & $\begin{array}{l}\text { Require temporal } \\
\text { temperature changes }\end{array}$ & $\begin{array}{l}\text { Temperature gradients } \\
\text { are necessary }\end{array}$ \\
& & Simple and robust & Low & High & Low \\
Cost & High & Reliance on external & Fabrication of high- \\
Specialty & Easy to implement & Poor energy & conversion ratio \\
& power source & quality thin film & & Does not need a \\
\end{tabular}

\section{RESEARCH METHOD}

Basically, a TEG consists of a two-layer structured, as shown in Figure 2, which are Al electrode coated with a mesoporous PDMS film at the top layer. However, at the bottom layer is another same size $\mathrm{Al}$ electrode coated gold ( $\mathrm{Au}$ ) deposit layer as shown in Figure 3. Mesoporous PDMS film is used in order to increase the contact surface area on top of increasing compressibility to generate high output power. In addition to that, the Au deposit enhances the stability due to their high oxidation resistance [25].

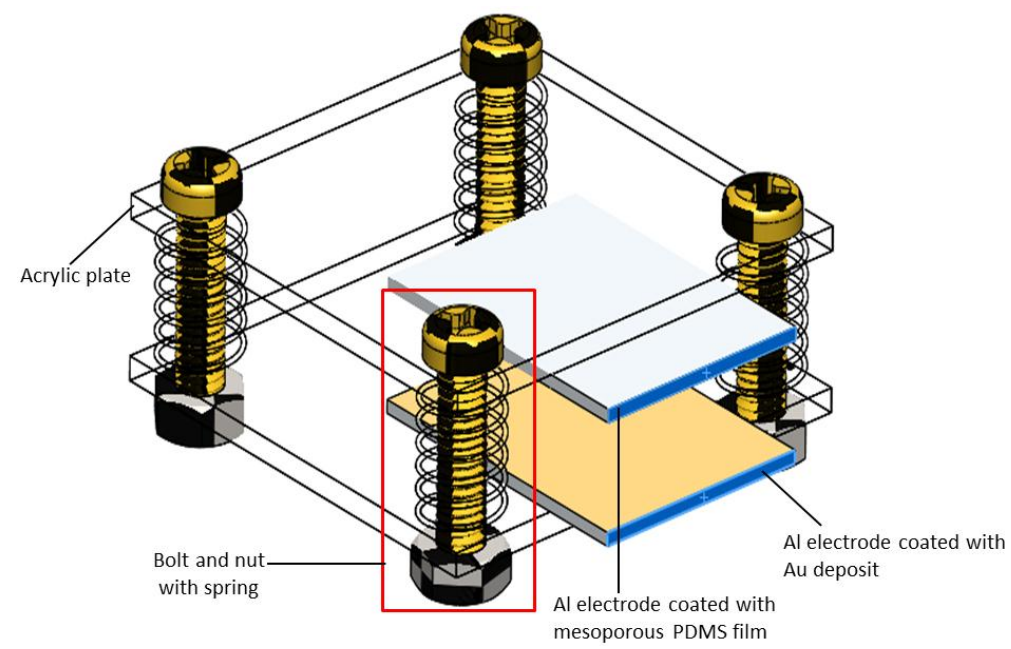

Figure 2. Design of TEG 


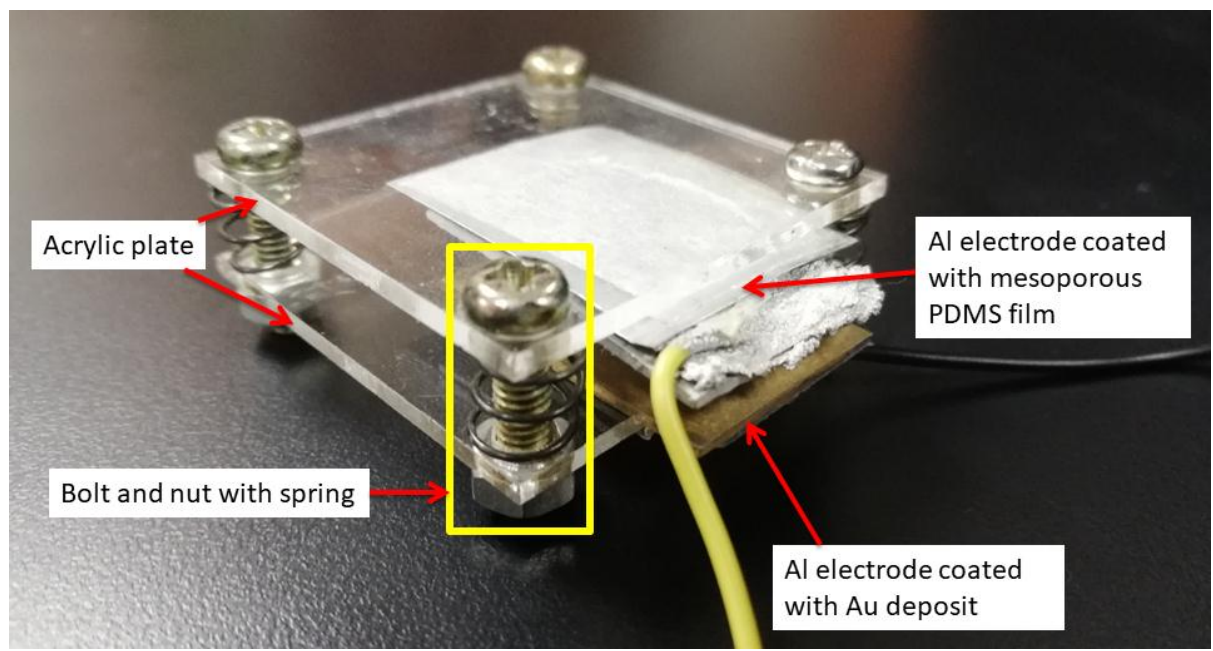

Figure 3. Actual design of TEG

The generation of voltage and current is due to the transfer of electrons through an external circuit whereby the process is in the sequence of initial state followed by first contact and separation respectively as shown in Figure 4. At initial state, positive and negative charges are distributed randomly in top Al electrode coated with mesoporous PDMS film as well as bottom Al electrode coated with Au deposit. When there is sufficient compression force acting on TEG, the mesoporous PDMS film is brought into contact with an $\mathrm{Au}$ deposit layer. This will cause the negative charges to accumulate at PDMS layer whereas positive charges accumulate at $\mathrm{Au}$ layer due to the electronegativity properties of the materials. As a result, positive charges are induced on the opposite surface of the PDMS layer whereas negative charges are induced on the opposite surface of Au layer, well known in Volta's electrophorus, known as the first electrostatic generator. Due to the difference in accumulation of positive and negative charges for both layers, a potential difference is created. As the compression force is withdrawn, the transfer of electrons will occur until it reaches an equilibrium state. Upon reaching charge equilibrium, electrons will stop transferring and there will be no more current and voltage generated. After the separation process, the process is back to initial state and will repeat as long as the compression force is still present. Therefore, in order for the TEG to generate power constantly, compression force needs to be applied repeatedly and hence the introduction of cyclic compression force on TEG in this project.

(a) Initial state

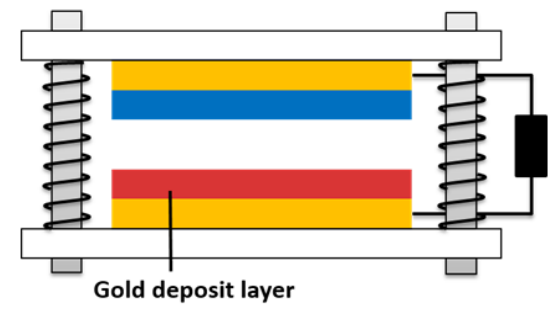

(b) First contact

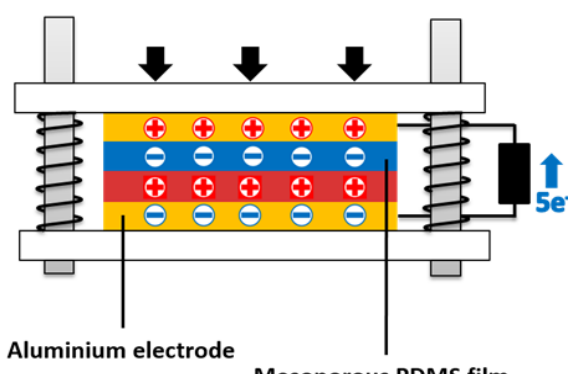

(c) Separation

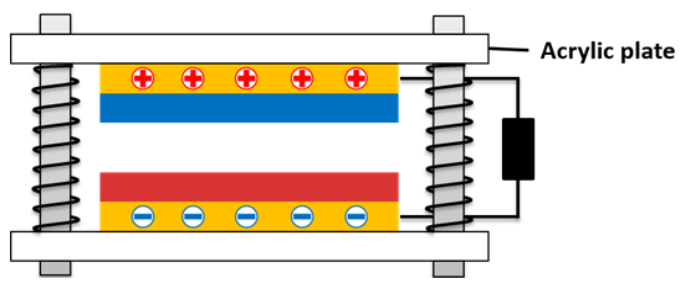

Figure 4. Operating principle for the generation of voltage and current output in TEG under external force 
In order to characterize the output performance for the suitable dielectric materials pair and operation mode, two experiments are set up whereby each TEG operation modes voltage output needs to be observed and recorded for different cyclic compression frequency and cyclic compression force applied on TEG. As for measurement, oscilloscope (GW Instek GDS-1072B digital storage oscilloscope) and digital multimeter (Keysight 34410A digital multimeter) are used for measuring the voltage and current output of TEG respectively. Figure 5 shows an experimental setup of triboelectric generator.

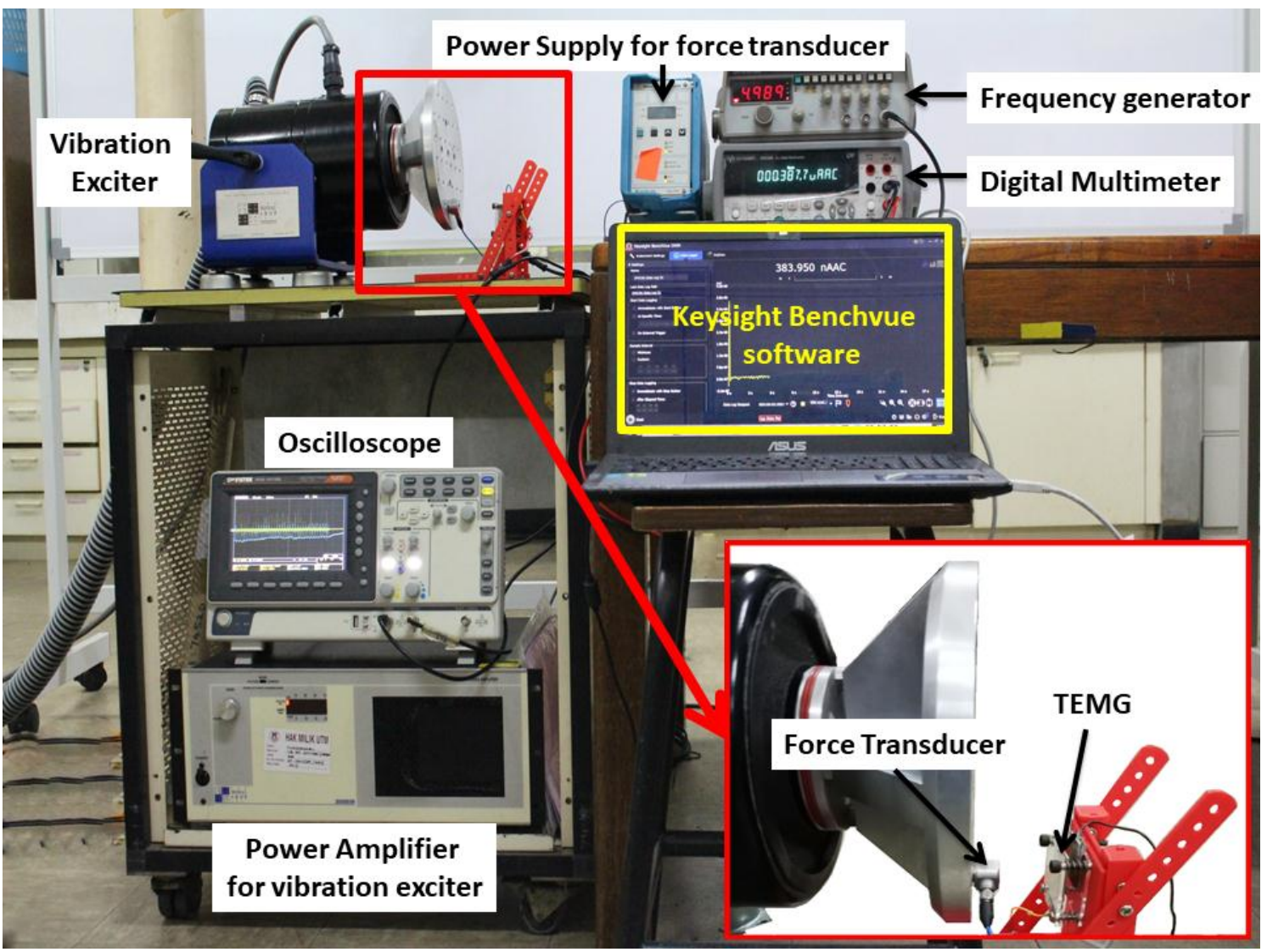

Figure 5. Experimental setup

\section{RESULTS AND DISCUSSION}

In this section, the characterization of TEG is analyzed by varying the frequency and cyclic compression force. To determine the characterization of TEG, a proper experimental work is done in two different experiment sets, namely frequency characterization and force characterization.

\subsection{Frequency characterization}

In frequency characterization, shown in Figure 6,15 $\mathrm{N}$ is applied to the TEG, whereas the voltage and current output are measured for ten different cyclic compression frequencies with $4 \mathrm{~cm}^{2}$ effective surface area under compression, the remaining area of $1.4 \mathrm{~cm}^{2}$ is used for measurement purposes where both $\mathrm{Al}$ electrodes are directly measured using oscilloscope paired with oscilloscope probe (HP-9100) of $1 \mathrm{M} \Omega$ input impedance. On the other hand, the current is measured in a series using a digital multimeter. Hence, the measured voltage is open-circuit voltage and the measured current is short-circuit current $\left(\mathrm{I}_{\mathrm{SC}}\right)$.

As the compression frequency increases, it is depicted that the $V_{\text {amp }}$ and $I_{S C}$ decreases based on Figures 6(a) and 6(b). This is due to the compressibility properties of triboelectric materials whereby the accumulation of electrons at PDMS layer and positive charges at Au layer are getting less saturated when the contact period between the two layers decreases as the compression frequency increases, thus leading to lower potential difference and less transfer of electrons. 

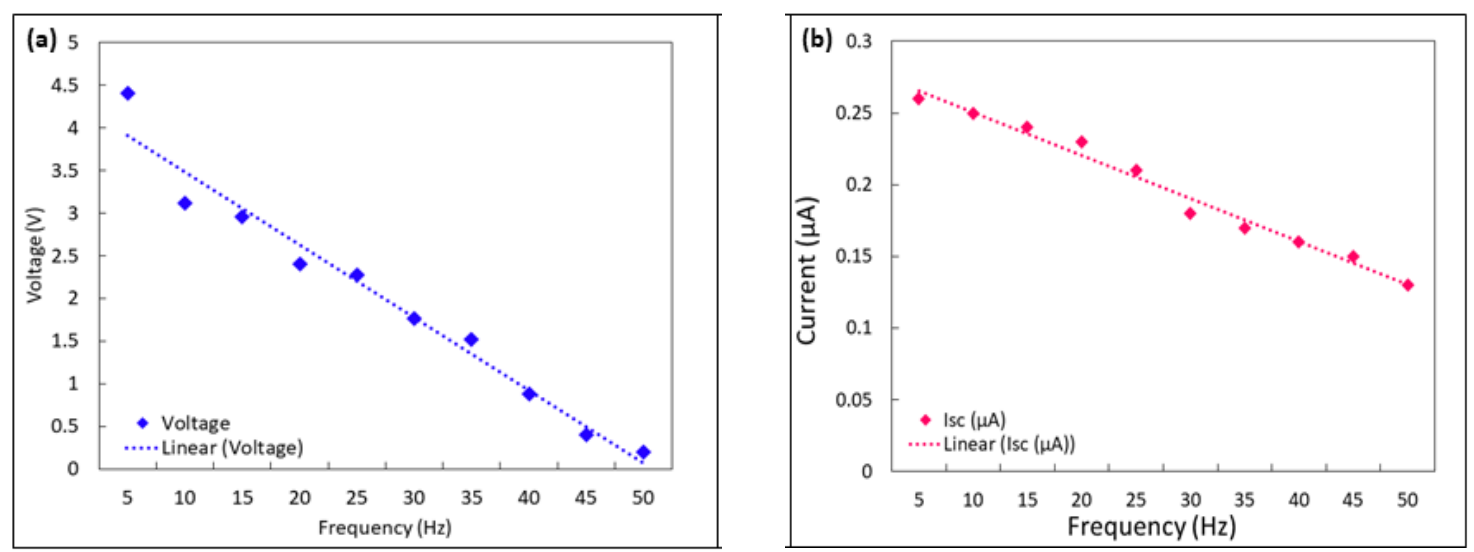

Figure 6. Frequency characterization, (a) $V_{a m p}$ output frequency, (b) $I_{s c}$ output frequency

\subsection{Force characterization}

In force characterization, the frequency is fixed at $5 \mathrm{~Hz}$ and simultaneously different force magnitude is being applied to TEG. From the experiment, the cyclic compression force cannot be controlled voluntarily. Hence, the force is controlled by increasing the power amplifier supplied to the compression mechanism. By using the force transducer on the compression mechanism, the force can be calculated depending on the magnitude of the voltage generated by the force transducer.

From Figure 7, $\mathrm{V}_{\text {amp }}$ remained at approximately $2 \mathrm{~V}$ when the force exceeded $22 \mathrm{~N}$. This means that the improvement in power output performance is considered to be less significant when the force is larger than $22 \mathrm{~N}$. However, the charge density in triboelectric materials approaches its maximum value as the force increases above $22 \mathrm{~N}$. Due to the compressibility properties of triboelectric materials, when the cyclic compression force acting on TEG increases, the charge density in triboelectric materials also increases. Therefore, it can be assumed that the cyclic compression force would generate effective power outputs as long as the force applied to TEG is above $22 \mathrm{~N}$

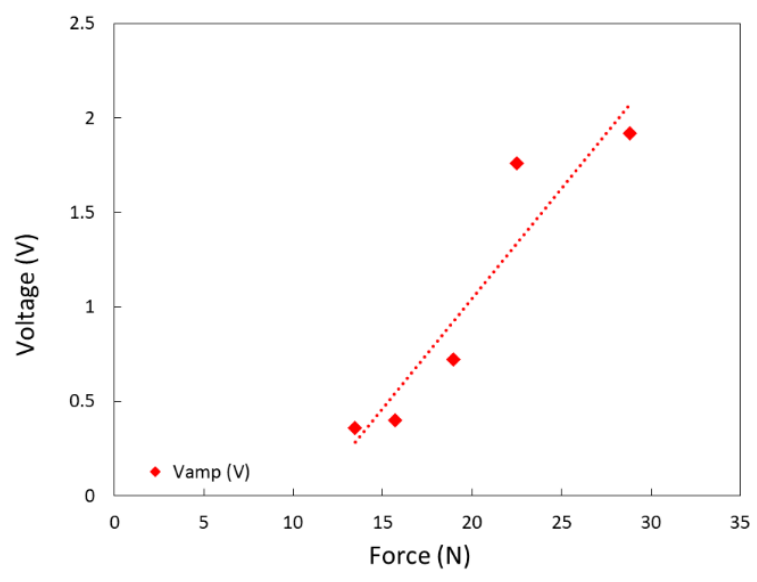

Figure 7. $\mathrm{V}_{\mathrm{amp}}$ output force

\section{CONCLUSION}

TEG using mesoporous PDMS and gold layer have been investigated. The power output per square meter is $2.85 \mathrm{~mW} / \mathrm{m}^{2}$ at the optimal frequency of $5 \mathrm{~Hz}$. By considering $2 \mathrm{~W}$ as the set minimum power consumption when powering up portable device such as charging cell phone, it will require $702 \mathrm{~m}^{2}$ of effective surface area of TEMG. Since the required effective surface area is too large and not suitable for portable application, hence further improvements need to be done to the TEMG system. The improvement need to be done for TEG is the power output improvement which can be achieved by magnifying the effective surface area of TEG while maintaining a small base area. 


\section{ACKNOWLEDGEMENTS}

This work has been supported by Contract Research Grant (CR 4C264) from Altech Industrial \& Engineering Supplies Sdn Bhd., Universiti Teknologi Malaysia (IIIG 01M69 \& CRG 05G35) and Ministry of Education Malaysia (FRGS 5F081).

\section{REFERENCES}

[1] J. D. Sterman, "System dynamics: Systems thinking and modelling for a complex world," ESD Working Paper Internal Symposium, Massachusetts Intitute of Technology, 2003.

[2] C. Zhang, W. Tang, C. Han, F. Fan, and Z. L. Wang, "Theoretical comparison, equivalent transformation, and conjunction operations of electromagnetic induction generator and triboelectric nanogenerator for harvesting mechanical energy," Adv. Mater., vol. 26, no. 22, pp. 3580-3591, 2014.

[3] Z. L. Wang and J. Song, "Piezoelectric nanogenerators based on zinc oxide nanowire arrays," Science., vol. 312, no. 5771, pp. 242-246, 2006.

[4] S. Xu, Y. Qin, C. Xu, Y. Wei, R. Yang, and Z. L. Wang, "Self-powered nanowire devices," Nat. Nanotechnol., vol. 5, no. 5, pp. 366-373, 2010.

[5] Z. L. Wang, G. Zhu, Y. Yang, S. Wang, and C. Pan, "Progress in nanogenerators for portable electronics," Mater. Today, vol. 15 , no. 12, pp. 532-543, 2012.

[6] L. Jiang, Y. Yang, R. Chen, G. Lu, R. Li, D. Li, M. S. Humayun, K K. Shung, J. Zhu, Y. Chen, and Q. Zhou, "Flexible piezoelectric ultrasonic energy harvester array for bio-implantable wireless generator," Nano Energy, vol. 56, pp. 216-224, 2019.

[7] D. Shen, "Piezoelectric energy harvesting devices for low frequency vibration applications," Thesis, Auburn University, p. 195, 2009.

[8] D. Ehre, E. Lavert, M. Lahav, and I. Lubomirsky, "Water freezes differently on positively and negatively charged surfaces of pyroelectric materials," Science, vol. 327, no. 5966, pp. 672-675, 2010.

[9] W. Punin, S. Maneewan, and C. Punlek, "Thermoelectric Generator for the Recovery of Energy from the LowGrade Heat Sources in Sugar Industry," International Journal of Power Electronics and Drive Systems, vol. 9, no. 4, pp.1565-1572, 2018.

[10] K. V. Selvan, M. N. Hasan, and M. S. Mohamed Ali, "Methodological reviews and analyses on the emerging research trends and progresses of thermoelectric generators," Int. J. Energy Res., vol. 43, no. 1, pp. 113-140, 2018.

[11] C. P. Chen, S. P. Koh, S. K. Tiong, J. D. Tan, and A. Y. C. Fong, "A heat waste recovery system via thermoelectric generator," Indonesian Journal of Electrical Engineering and Computer Science, vol. 16, no. 2, pp. 586-590, 2019.

[12] N. Baatar and S. Kim, "A thermoelectric generator replacing radiator for internal combustion engine vehicles," TELKOMNIKA (Telecommunication, Computing, Electronics and Control), vol. 9, no. 3, pp. 523-530, 2011.

[13] M. N. Hasan, H. Wahid, N. Nayan, and M. S. M. Ali, "Inorganic thermoelectric materials: A review," International Journal of Energy Research, Early view, pp. 1-53, 2020.

[14] K. V Selvan, T. Rehman, T. Saleh, and M. S. M. Ali, "Copper-cobalt thermoelectric generators: Power improvement through optimized thickness and sandwiched planar structure," IEEE Trans. Electron Devices, vol. 66, no. 8, pp. 3459-3465, 2019.

[15] S. J. Kim, J. H. We, and B. J. Cho, "A wearable thermoelectric generator fabricated on a glass fabric," Energy and Environ. Sci., vol. 7, no. 6, pp. 1959-1965, 2014.

[16] H. Chen, Y. Song, X. Cheng, and H. Zhang, "Self-powered electronic skin based on the triboelectric generator," Nano Energy, vol. 56, pp. 252-268, 2019.

[17] K. V. Selvan, M. N. Hasan, and M. S. Mohamed Ali, "State-of-the-art reviews and analyses of emerging research findings and achievements of thermoelectric materials over the past years," J. Electron. Mater., vol. 48, no. 2, pp. 745-777, 2019.

[18] F. R. Fan, Z. Q. Tian, and Z. Lin Wang, "Flexible triboelectric generator," Nano En., vol. 1, no. 2, pp. 328-334, 2012.

[19] J. Chen, G. Zu, W. Yang, Q. Jing, P. Bai, Y. Yang, T-C. Hou, and Z. L. Wang, "Harmonic-resonator-based triboelectric nanogenerator as a sustainable power source and a self-powered active vibration sensor," Adv. Mater., vol. 25, no. 42, pp. 6094-6099, 2013.

[20] G. Sebald, E. Lefeuvre, and D. Guyomar, "Pyroelectric energy conversion: Optimization principles," IEEE Trans. Ultrason. Ferroelectr. Freq. Control, vol. 55, no. 3, pp. 538-551, 2008.

[21] S. Wang, S. Niu, J. Yang, L. Lin, and Z. L. Wang, "Quantitative measurements of vibration amplitude using a contact-mode freestanding triboelectric nanogenerator," ACS Nano, vol. 8, no. 12, pp. 12004-12013, 2014.

[22] G. Zhu, Z-H. Lin, Q. Jing, P. Bai, C. Pan, Y. Yang, Y. Zhou, and Z. L Wang, "Toward large-scale energy harvesting by a nanoparticle-enhanced triboelectric nanogenerator," Nano Lett., vol. 13, no. 2, pp. 847-853, 2013.

[23] Y. Yang, Y. S. Zhou, H. Zhang, Y. Liu, S. Lee, and Z. L. Wang, "A single-electrode based triboelectric nanogenerator as self-powered tracking system," Adv. Mater., vol. 25, no. 45, pp. 6594-6601, 2013.

[24] S. Wang, L. Lin, Y. Xie, Q. Jing, S. Niu, and Z. L. Wang, "Sliding-triboelectric nanogenerators based on in-plane charge-separation mechanism," Nano Lett., vol. 13, no. 5, pp. 2226-2233, 2013.

[25] Y. Su, X. Wen, G. Zhu, J. Yang, J. Chen, P. bai, Z. Wu, Y. Jiang, and Z. L. Wang, "Hybrid triboelectric nanogenerator for harvesting water wave energy and as a self-powered distress signal emitter," Nano Energy, vol. 9, pp. 186-195, 2014. 


\section{BIOGRAPHIES OF AUTHORS}

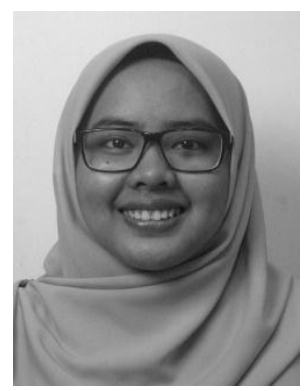

Mariatul Rawdhah Ahmad Fuaad received the B.Sc. Degree in Science Instrumentation from Universiti Putra Malaysia, Serdang, Malaysia in 2018. She is currently pursuing her Masters Degree in Electrical Engineering at Universiti Putra Malaysia.

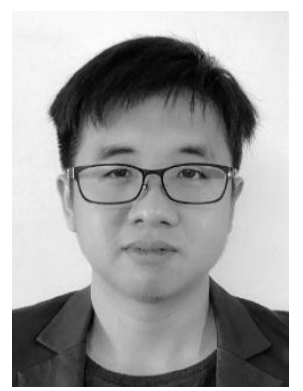

Lee Chang Yang received the B.Eng. degree in Mechatronics engineeting from Universiti Teknologi Malaysia, Skudai, Malaysia, in 2019 with a focus on triboelectric generator.

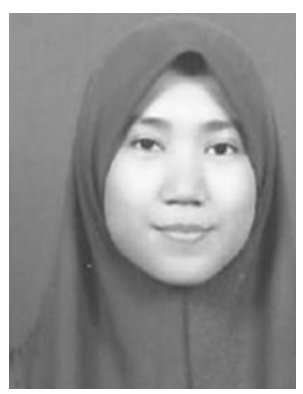

Farah Afiqa Mohd Ghazali received B.Eng. degree in Mechanical engineering from Universiti Teknologi Malaysia, Skudai, Malaysia in 2016. She is currently pursuing her Masters degree in Electrical Engineering at Universiti Teknologi Malaysia. Her research interes is in the field of MEMS sensor and actuators

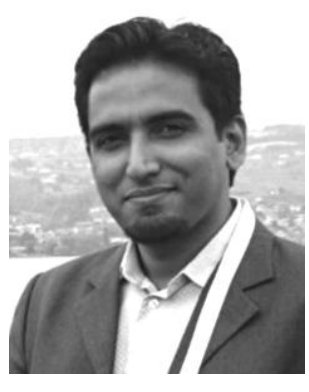

Mohamed Sultan Mohamed Ali received the B.Eng. and M.Eng. degrees in electrical engineering from Universiti Teknologi Malaysia, Skudai, Johor, Malaysia, in 2006 and 2008, respectively, and the Ph.D. degree in electrical and computer engineering from the Department of Electrical and Computer Engineering, The University of British Columbia, Vancouver, BC, Canada, in 2012. From 2001 to 2007, he held various engineering positions at Flextronics International Ltd. and Jabil Circuit, Inc. He is currently an Associate Professor in the Faculty of Electrical Engineering, Universiti Teknologi Malaysia. Dr. Mohamed Sultan serves as a consultant for Flextronics Malaysia in the failure analysis division and is actively involved in many industrial projects. He was awarded Chartered Engineer (C.Eng.) and Professional Engineer (P.Eng.) by United Kingdom Engineering Council and Board of Engineers Malaysia, respectively. Dr. Mohamed Sultan is also a Senior Member of IEEE (SMIEEE). Dr. Mohamed Sultan has published over 100 technical papers in MEMS and related fields. He has also served as the editor of 5 books in Mechatronics. His research interests are in the areas of MEMS, nanotechnology, and also in micro/ nanofabrication technologies, including wireless microdevices, integration of microstructures, and microrobotics. 